\title{
Plasma extracellular vesicles as a source of biomarkers in traumatic brain injury
}

\author{
Ross C. Puffer, MD,, Luz M. Cumba Garcia, MS, ${ }^{2,3}$ Benjamin T. Himes, MD,, Mi-Yeon Jung, PhD,1 \\ Frederic B. Meyer, MD, ${ }^{1}$ David O. Okonkwo, MD, PhD, ${ }^{4}$ and lan F. Parney, MD, PhD ${ }^{1,2}$ \\ Departments of ${ }^{1}$ Neurological Surgery and ${ }^{2}$ mmunology, Mayo Clinic, Rochester; ${ }^{3}$ Mayo Clinic Graduate School of Biomedical \\ Sciences, Rochester, Minnesota; and ${ }^{4}$ Department of Neurosurgery, University of Pittsburgh, Pennsylvania
}

\begin{abstract}
OBJECTIVE The objective of this study was to isolate extracellular vesicles (EVs) from plasma in a cohort of patients with traumatic brain injury (TBI) and analyze their contents for novel biomarkers that could prove useful for rapid diagnosis and classification of brain injury during initial evaluation.

METHODS Plasma EVs were isolated by serial ultracentrifugation from patients with $\operatorname{TBI}(n=15)$ and healthy controls $(n=5)$. Samples were obtained from the TRACK-TBI biorepository (2010-present). Size and concentration were determined by nanoparticle tracking. Glial fibrillary acidic protein (GFAP) concentration was determined in EV protein. EV RNA was isolated and deep sequencing of short noncoding RNA was performed.

RESULTS Plasma EVs are physically similar but contained approximately 10 times more GFAP in TBI patients with altered consciousness than patients and controls with normal consciousness. Eleven highly differentially expressed microRNAs (miRNAs) were identified between these groups. Genes targeted by these miRNAs are highly associated with biologically relevant cellular pathways, including organismal injury, cellular development, and organismal development. Multiple additional coding and noncoding RNA species with potential biomarker utility were identified.

CONCLUSIONS Isolating plasma EVs in patients with TBI is feasible. Increased GFAP concentration-a validated plasma TBI marker-in EVs from TBI patients with altered consciousness, along with differential expression of multiple miRNAs targeting TBI-relevant pathways, suggests that EVs may be a useful source of TBI biomarkers. Additional evaluation in larger patient cohorts is indicated.

https://thejns.org/doi/abs/10.3171/2020.4.JNS20305
\end{abstract}

KEYWORDS extracellular vesicle; biomarker; microRNA; plasma; diagnostic; traumatic brain injury

$\mathrm{E}$ XTRACELlular vesicles (EVs) are membrane-bound particles released by the majority of human cells, including cells within the CNS., ${ }^{1,2}$ They contain DNA, messenger RNA (mRNA), noncoding RNA, and proteins that are released into the microenvironment, and play key roles in cell-to-cell communication between neurons, glia, and endothelial cells for complex functions including moderation of the blood-brain barrier. ${ }^{3-5}$ EVs are a heterogeneous group composed of microvesicles $(>100$ $\mathrm{nm})$ and smaller exosomes $(<100 \mathrm{~nm})$ that are generated through distinct cellular pathways and have distinct cargo. EVs have generated significant interest in neurooncology; tumor-derived EVs shape the microenvironment by downregulating the immune response and upregulating angiogenic factors, among other complex actions..$^{3-6}$
Severe traumatic brain injury (TBI) affects more than 200,000 individuals annually in the US and is a leading cause of mortality in men under 35 years of age. ${ }^{7}$ Mild TBI is underreported but may be as much as 10 times more common than severe TBI, and is increasingly recognized as a contributor to chronic neurological dysfunction. ${ }^{8,9}$ Plasma biomarkers in TBI have generated substantial excitement recently with the identification of multiple brainspecific molecules (glial fibrillary acidic protein [GFAP], ubiquitin carboxy-terminal hydrolase L1 [UCH-L1], neurofilament light, tau) in the blood of patients with TBI and recent FDA approval of a device (Brain Trauma Indicator, Banyan Biomarkers) for rapid measurement of GFAP and UCH-L1 levels in plasma..$^{10}$ These biomarkers are used to help clarify TBI diagnosis and prognosis, particularly

ABBREVIATIONS EV = extracellular vesicle; GCS = Glasgow Coma Scale; GFAP = glial fibrillary acidic protein; lincRNA = long intervening noncoding RNA; miRNA = microRNA; $m R N A$ = messenger RNA; rRNA = ribosomal RNA; snoRNA = small nucleolar RNA; snRNA = small nuclear RNA; TBI = traumatic brain injury; TRACK-TBI = Transforming Research and Clinical Knowledge in Traumatic Brain Injury; UCH-L1 = ubiquitin carboxy-terminal hydrolase L1.

SUBMITTED January 31, 2020. ACCEPTED April 28, 2020.

INCLUDE WHEN CITING Published online July 24, 2020; DOI: 10.3171/2020.4.JNS20305. 
in mild TBI. EVs have not yet been extensively studied in TBI, although one small study has identified a higher number of EVs in CSF of two subjects with severe TBI (Glasgow Coma Scale [GCS] score 3-8) compared to normal controls, and further isolated both GFAP and UCHL1 in higher quantities in the TBI subjects compared to controls. ${ }^{11}$ While these findings are intriguing, obtaining similar results in plasma would be preferable to CSF as plasma is much more readily available. EVs provide a potential source of brain-specific biomarkers that can be isolated from peripheral blood.

EVs in peripheral blood provide a lipid membranebound container of DNA, RNA, and proteins that are protected from circulating degradation enzymes, and therefore may provide a snapshot of the microenvironment in specific organ systems shortly before the peripheral blood sample is taken. This makes plasma EVs potentially an intriguing source of brain-specific biomarkers that may provide real-time insight into the cellular microenvironment during the initial brain injury phase, as well as during both short- and long-term recovery. Beyond diagnosis and prognosis, EVs have potential therapeutic applications as well, but the exact source of therapeutic EVs in TBI and what, if any, modification would be necessary to render them therapeutic is yet unclear. In this pilot study, we sought to assess the potential of plasma EVs as a biomarker source in TBI by demonstrating the feasibility of isolating EVs, characterizing their size and frequency, and determining the presence of both established (GFAP) and novel (short noncoding RNA) TBI biomarkers within them, comparing plasma samples from TBI patients to healthy controls. A difference in RNA expression profiles contained within the EVs of TBI patients compared to healthy controls was hypothesized.

\section{Methods \\ Study Population}

This study was a joint analysis between the University of Pittsburgh and the Mayo Clinic. IRB approval was obtained at both institutions. Subject plasma samples (approximately $500 \mu \mathrm{l}$ each) were selected from the TRACKTBI (Transforming Research and Clinical Knowledge in Traumatic Brain Injury) biorepository from the University of Pittsburgh. ${ }^{12}$ Acquisition and sharing of these samples was approved by the University of Pittsburgh IRB. This project was reviewed by the Mayo Clinic IRB and believed to be IRB-exempt at is was secondary research on biopspecimens for which consent is not required and the identity of human subjects cannot be readily ascertained by the investigators. A total of 20 plasma samples were obtained: 15 samples were obtained after TBI, and 5 samples were obtained from healthy controls. Patients with complicated mild (GCS score 13-15 with evidence of injury on CT scan), moderate (GCS score 9-12), and severe (GCS score 3-8) TBI were included. Given the pilot nature of this study, attempts were made to select patients with a wide range of injury mechanisms accompanying all severities of TBI as well as from uninjured, healthy controls. Demographic and injury information is listed in Table 1.

\section{Isolation of EVs, Western Blot, and GFAP Analysis}

EVs within plasma were isolated using serial density gradient ultracentrifugation per our established protocol. ${ }^{13}$ Concentrated EVs were then analyzed via nanotracker particle analysis using NanoSight (Malvern, NanoSight NS300). NanoSight allows for characterization of EVs, including both size and concentration within a given sample. As a part of this protocol for isolating EVs, a western blot analysis was performed to identify common EV proteins, confirming the presence of EVs in the sample. For western blot analysis, proteins were separated by electrophoresis on $4 \%-20 \%$ sodium dodecyl sulfate-polyacrylamide gel. Following membrane transfer, the proteins were probed using the following antibodies: anti-Alix (Cell Signaling, no. 2171S, mouse monoclonal), anti-CD9 (Cell Signaling, no. 13174S, rabbit monoclonal), anti-CD63 (Santa Cruz Biotechnology, no. sc-5275, mouse monoclonal), and antiHSP90 (Cell Signaling, no. 4874S, rabbit polyclonal). Secondary antibody was horseradish peroxidase-conjugated goat antirabbit or goat antimouse (Jackson ImmunoResearch, no. 115-035-003). Detection was conducted using enhanced chemiluminescence (ThermoFisher Scientific, no. 32106). For GFAP analysis, EVs were sonicated to break their membranes. GFAP expression was then tested in all samples via ELISA using a commercially available GFAP kit (abcam, no. ab223867) per the manufacturer's instructions.

\section{MicroRNA Analysis}

Short noncoding RNA was isolated from samples using an miRNAeasy kit and QiaCube (QIAGEN). Preliminary quality assessment was performed using NanoDrop (ThermoFisher). Samples then underwent next-generation sequencing using the Ilumina MiSeq 2000 with NebNext multiplex oligos for Illumina adapters (adapter sequence: AGATCGGAAGAGCACACGTCT). Analysis of short noncoding RNA sequencing was performed in collaboration with the Bioinformatics Core of the Mayo Clinic Center for Individualized Medicine. Sequencing data were analyzed using the OASIS 2.0 platform with the reference genome HG38; this investigated 28,263 unique RNA entities covering microRNA (miRNA), Piwi-interacting RNA, small nucleolar RNA (snoRNA), small nuclear RNA (snRNA), and ribosomal RNA (rRNA) species. Differential expression analysis was used to identify differences in miRNA expression between groups.

\section{Statistical Analysis}

Descriptive statistics with medians, interquartile ranges, and proportions were used to describe continuous and categorical variables, respectively. Biomarker levels were treated as continuous data. The Mann-Whitney U-test was used to assess for between-group differences for continuous variables. All statistical analysis was performed with JMP software.

\section{Results}

EVs were isolated from 15 TBI subjects, including 6 with CT-positive mild TBI (GCS score 13-15), 3 with CTpositive moderate TBI (GCS score 9-12), and 6 with CT- 
positive severe TBI (GCS score 3-8), as well as 5 healthy control subjects with no history of TBI or neurodegeneration. Given the relatively small sample size, subjects were divided into those who presented with an altered level of consciousness (GCS score $\leq 14$ ) and those with a normal level of consciousness (GCS score of 15 and healthy controls). Demographic and injury information is listed in Table 1.

\section{Isolation of EVs and GFAP Analysis}

EVs were successfully isolated in all samples analyzed. EV size and concentration were available for each sample (Fig. 1). EV size, concentration, GFAP concentration, and RNA yield for the entire cohort are shown in Table 2. There were no significant differences in EV size, concentration, or RNA yield between normal controls/TBI patients with normal consciousness (GCS score $=15$ ) and TBI patients with altered consciousness (GCS score $\leq 14$; Fig. 2). In contrast, subsequent analysis of GFAP yield by ELISA demonstrated a significantly higher GFAP concentration in subjects with an altered level of consciousness (GCS score $\leq 14$ ) as compared to those with normal GCS scores (mean GFAP concentration [ \pm standard error] $2204.2 \mathrm{pg} / \mathrm{ml} \pm 1067.2 \mathrm{pg} / \mathrm{ml}$ vs $207.8 \mathrm{pg} / \mathrm{ml} \pm 270.8 \mathrm{pg} /$ $\mathrm{ml}, \mathrm{p}=0.02$; Fig. 2).

\section{Short Noncoding RNA Sequencing}

RNA was successfully isolated from all samples, and RNA yields from the isolated EVs are listed in Table 2. Short noncoding RNA sequencing for plasma EVs from TBI patients and healthy controls was analyzed on the Illumina HiSeq 2500 platform and processed via our CAPmiRSeq pipeline. This allowed unbiased identification of known and novel short noncoding RNA species beyond simply miRNAs. Differential expression analysis was performed for miRNA sequences identified with thresholds of $\log 2$-fold change $(0.7)$ and a $\mathrm{p}$ value $<0.1$, given the low number of samples with widely varying demographics, injury mechanisms, and presentation GCS scores. Using these criteria, trends for 9 highly upregulated miRNAs and 2 highly downregulated miRNAs were identified in TBI patients with an altered level of consciousness, as compared to TBI patients and healthy controls with a normal level of consciousness (Fig. 3A, Table 3). These reached statistical significance $(\mathrm{p} \leq 0.05)$ for 7 of the 11 individual miRNA sequences identified. Furthermore, Ingenuity pathway analysis of highly differentially expressed miRNAs among all TBI patients relative to controls identified top disease and disorder pathways targeted, including cancer, gastrointestinal disease, and organismal injuries/ abnormalities, while the top molecular and cellular pathways identified included cell death/survival, cell-to-cell signaling, and cellular assembly/organization (Table 4).

miRNAs are well characterized in existing databases, which facilitated their analysis for the present study. However, they actually made up a minority of RNA species found within plasma EVs (Fig. 3B). The majority of the RNA found was protein-coding mRNA. Due to the specific parameters of our sequencing paradigm, which was aimed at short noncoding RNA, we were unable to mean-
TABLE 1. Cohort demographics, injury mechanisms, and presentation GCS scores

\begin{tabular}{|c|c|c|c|}
\hline Cohort & Age (yrs), Sex & Injury Mechanism & $\begin{array}{l}\text { Presentation } \\
\text { GCS Score }\end{array}$ \\
\hline \multicolumn{4}{|l|}{ TBI patients } \\
\hline TR-1345 & $22, \mathrm{~F}$ & MVC & 15 \\
\hline TR-1134 & $41, \mathrm{M}$ & MVC & 15 \\
\hline TR-1146 & $32, \mathrm{M}$ & Fall & 15 \\
\hline TR-1395 & $46, M$ & MVC & 14 \\
\hline TR-1178 & $25, M$ & MVC & 14 \\
\hline TR-1123 & $72, \mathrm{M}$ & Fall & 13 \\
\hline TR-1161 & $33, \mathrm{M}$ & Assault & 11 \\
\hline TR-1252 & $24, \mathrm{M}$ & MVC & 10 \\
\hline TR-1082 & $55, F$ & Fall & 9 \\
\hline TR-1330 & $35, \mathrm{M}$ & Other & $6 \mathrm{~T}$ \\
\hline TR-1174 & $59, \mathrm{M}$ & MVC & 3 \\
\hline TR-1086 & $56, F$ & Fall & 3 \\
\hline TR-1219 & $45, M$ & MVC & 3 \\
\hline TR-1233 & $30, \mathrm{M}$ & MVC & 3 \\
\hline TR-1159 & $23, \mathrm{M}$ & MVC & 3 \\
\hline \multicolumn{4}{|c|}{ Healthy controls } \\
\hline HC-1054 & $25, \mathrm{M}$ & - & 15 \\
\hline HC-1052 & $31, \mathrm{M}$ & - & 15 \\
\hline HC-1015 & $40, M$ & - & 15 \\
\hline HC-1019 & $53, \mathrm{~F}$ & - & 15 \\
\hline HC-1058 & $63, \mathrm{M}$ & - & 15 \\
\hline
\end{tabular}

MVC = motor vehicle collision

ingfully quantify the protein-coding transcripts found in the present data. Nevertheless, they are present in abundance. Similarly, multiple additional species of short noncoding RNA were found, including snoRNA, snRNA, rRNA, and long intervening noncoding RNA (lincRNA).

\section{Discussion}

In this pilot analysis of plasma EV isolation and sampling in TBI, we demonstrated successful EV isolation from plasma samples drawn at the time of initial hospital evaluation. Plasma EVs from TBI patients with altered consciousness (GCS score $\leq 14$ ) are physically similar to plasma EVs from TBI patients and normal donors with normal consciousness (GCS score $=15$ ) but are markedly enriched for GFAP, an established plasma biomarker in TBI. EVs are known to be rich sources of nucleic acids, proteins, and metabolites. The presence of GFAP in plasma EV suggests that they also likely harbor transcriptomic, proteomic, and metabolomic signatures potentially unique to TBI diagnosis that could be isolated and analyzed. As an example of this potential, our deep sequencing of short noncoding RNA identified 11 highly differentially expressed miRNA sequences between TBI patients with altered consciousness and TBI patients/ healthy controls with normal consciousness. Furthermore, these miRNAs targeted genes associated with highly bio- 

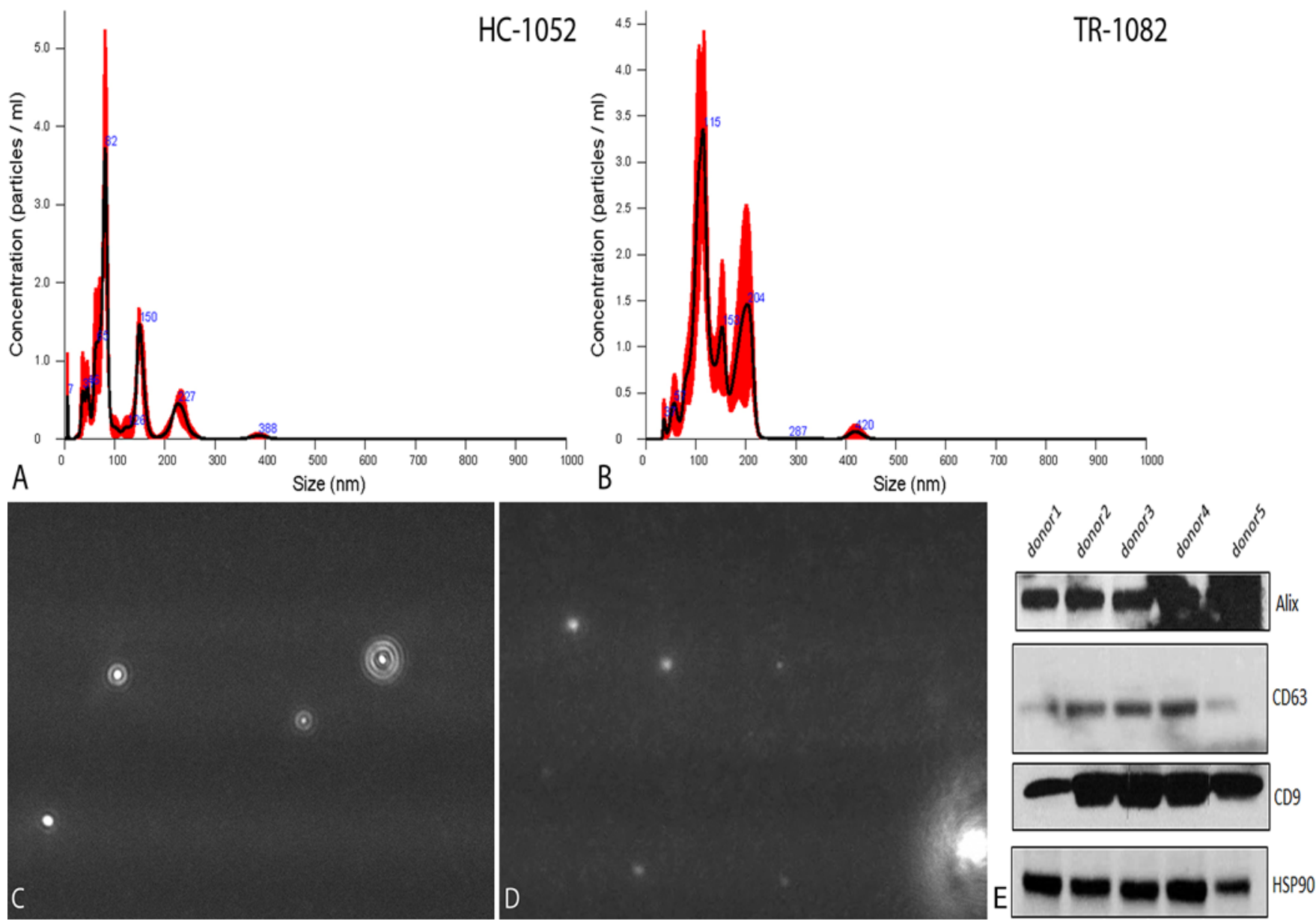

FIG. 1. Nanoparticle tracking analysis. Representative histograms (A and B) and photomicrographs (C and D) of EV analysis in a healthy control (A and $C$ ) and TBI subject (B and D). The histograms in both the healthy control $(A)$ and TBI patient (B) demonstrate a substantial exosome peak $(<100 \mathrm{~nm})$ and multiple smaller microvesicle peaks $(>100 \mathrm{~nm})$. Original magnification $\times 20$. Western blot $(\mathrm{E})$ shows expression of common EV-associated proteins in EVs isolated with this protocol. Figure is available in color online only.

logically relevant pathways in trauma (e.g., organismal injury and cell death/survival). These findings suggest that brain-specific EVs are released shortly after TBI, resulting in an altered level of consciousness, and that these EVs are obtainable via peripheral blood draws during initial evaluation in the emergency department. Given the highly specific and measurable contents of the EVs, their isolation and analysis provide opportunities for the identification of potentially novel biomarkers that can be correlated with injury severity, prognosis, or response to therapeutic interventions.

It is important to note that there is a constant stream of EVs circulating in peripheral blood from nearly every cell in the body, even in a healthy, resting state. ${ }^{14}$ EVs are not solely produced by the CNS after trauma, and likely make up only a small fraction of the total circulating EV burden at any given time, even after trauma. This is consistent with our findings, given that there was no significant difference between EV concentration or size in any of the trauma subjects compared to each other or healthy controls. Therefore, it is likely that multiple organ systems, not just the CNS, change their EV release patterns after trauma. Identification of brain-specific EVs is challenging and remains under investigation. For this analysis, we chose to analyze bulk plasma EVs (not brain-derived EVs specifically) and demonstrated that GFAP, a brain-specific biomarker released after trauma, is increased in plasma EVs following TBI with altered consciousness. This suggests that, even in bulk plasma EVs, relevant CNS biomarkers are increased following TBI. It also suggests that EVs from peripheral blood are another quantifiable source of GFAP in cases of TBI, perhaps having uses in the initial evaluation and diagnosis of TBI. . $^{8,10,12}$

Importantly, the same bulk plasma EVs enriched for the brain-specific biomarker GFAP also contained miRNA signatures that significantly differed between subjects with an altered level of consciousness as compared to normal level of consciousness at the time of peripheral blood draw. These miRNAs have hundreds of downstream targets within the transcriptome and represent novel biomarkers that may be useful for diagnosis, prognosis, and management in TBI. Our pathway analysis demonstrated that these 
TABLE 2. MV/E concentration, mean size, GFAP concentration, and RNA concentration

\begin{tabular}{|c|c|c|c|c|}
\hline Study Subject (GCS Score) & MV/E Concentration (particles/ml) & MV/E Size (nm) & GFAP Concentration (pg/ml) & RNA Concentration ( $\mu \mathrm{g} / \mathrm{ml})$ \\
\hline \multicolumn{5}{|l|}{ TBI } \\
\hline TR-1345 (15) & $36,300,000,000$ & 85.2 & 2166.68 & 61.4 \\
\hline TR-1134 (15) & $93,900,000,000$ & 68.6 & Undetectable & 37.1 \\
\hline TR-1146 (15) & $145,000,000,000$ & 89.5 & Undetectable & 30.7 \\
\hline TR-1395 (14) & $12,100,000,000$ & 107.1 & 3489.56 & 42.4 \\
\hline TR-1178 (14) & $89,400,000,000$ & 67.7 & 136.4 & 46 \\
\hline TR-1123 (13) & $13,400,000,000$ & 124.9 & 2164.03 & 57.6 \\
\hline TR-1161 (11) & $282,000,000,000$ & 71.7 & Undetectable & 33 \\
\hline TR-1252 (10) & $18,700,000,000$ & 137.2 & 466.6 & 54.7 \\
\hline TR-1082 (9) & $20,100,000,000$ & 139 & Undetectable & 62.4 \\
\hline TR-1330 (6T) & $8,480,000,000$ & 92.7 & 1381.7 & 47.6 \\
\hline TR-1174 (3) & $11,600,000,000$ & 154.1 & Undetectable & 76.8 \\
\hline TR-1086 (3) & $32,200,000,000$ & 105 & 221.99 & 32.3 \\
\hline TR-1219 (3) & $28,400,000,000$ & 85.1 & 3834.9 & 42.6 \\
\hline TR-1233 (3) & $31,700,000,000$ & 106.6 & 13122.5 & 86.6 \\
\hline TR-1159 (3) & $11,300,000,000$ & 108.3 & 1633.1 & 16 \\
\hline \multicolumn{5}{|l|}{ Healthy control } \\
\hline HC-1054 (15) & $64,200,000,000$ & 64.6 & Undetectable & 55.9 \\
\hline HC-1052 (15) & $12,200,000,000$ & 114 & Undetectable & 65.3 \\
\hline HC-1015 (15) & $45,800,000,000$ & 114.8 & Undetectable & 40.2 \\
\hline HC-1019 (15) & $53,400,000,000$ & 79.1 & Undetectable & 53.2 \\
\hline HC-1058 (15) & $55,200,000,000$ & 97.8 & Undetectable & 17 \\
\hline \multicolumn{5}{|l|}{ Analysis } \\
\hline Mean altered GCS score & $4.7 e+10 \pm 2.2 e+10$ & $108.3 \pm 7.7$ & $2204.2 \pm 1067.2$ & $49.8 \pm 5.6$ \\
\hline Mean normal GCS score & $6.3 e+10 \pm 1.4 e+10(N S)$ & $89.2 \pm 6.7$ (NS) & $207.8 \pm 270.8(p=0.02)$ & $45.1 \pm 5.9(\mathrm{NS})$ \\
\hline
\end{tabular}

MV $/ E$ = microvesicles/exosomes; $\mathrm{NS}=$ nonsignificant.

up- and downregulated miRNAs play a role in cell maintenance, assembly, and death, as well as nervous system function. Further analysis could focus on protein-coding mRNA, snoRNA, snRNA, rRNA, and a wide range of as yet unknown protein signatures contained within these EVs. Each sample is a snapshot in time, and the signatures could change over time, in response to either further insult (persistently raised intracranial pressure, decreased cere- bral blood flow, poor oxygen delivery, etc.) or therapeutic maneuvers (medications and/or surgical intervention). The signatures obtained and analyzed from EVs could be a technique to monitor function and recovery of the CNS at a cellular level using the body's own cell-to-cell signaling mechanisms. This area of research remains in relative infancy, and significant work is required to better understand the factors surrounding EV release after many types
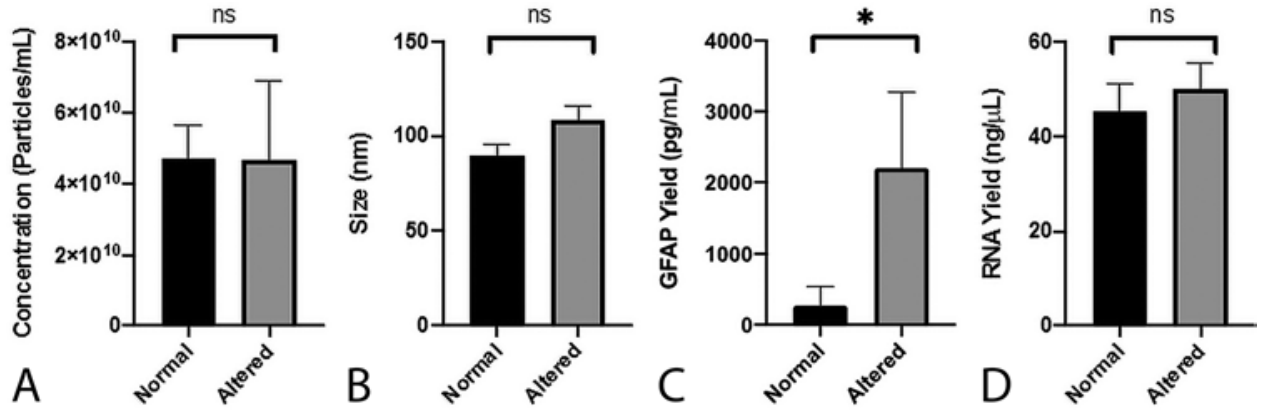

FIG. 2. EV characteristics. Comparison between TBI patients and normal controls with normal consciousness (GCS score $=15)$ versus TBI patients with altered consciousness (GCS score $\leq 14$ ) for EV concentration (A), EV size (B), EV GFAP yield (C), and EV RNA yield (D). Note that GFAP yield is significantly higher in TBI patients with altered consciousness but no significant differences are seen in EV concentration, size, or RNA yield. Values represent mean \pm standard error. ${ }^{*} p<0.05$. 


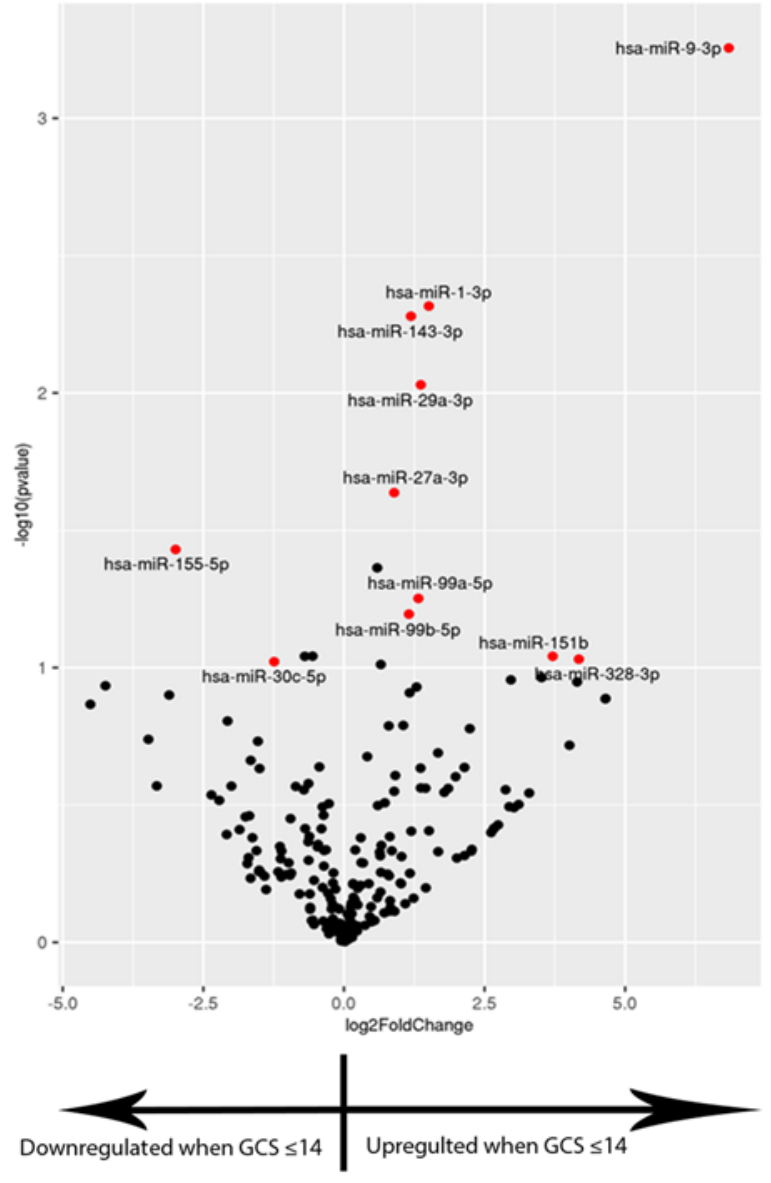

A

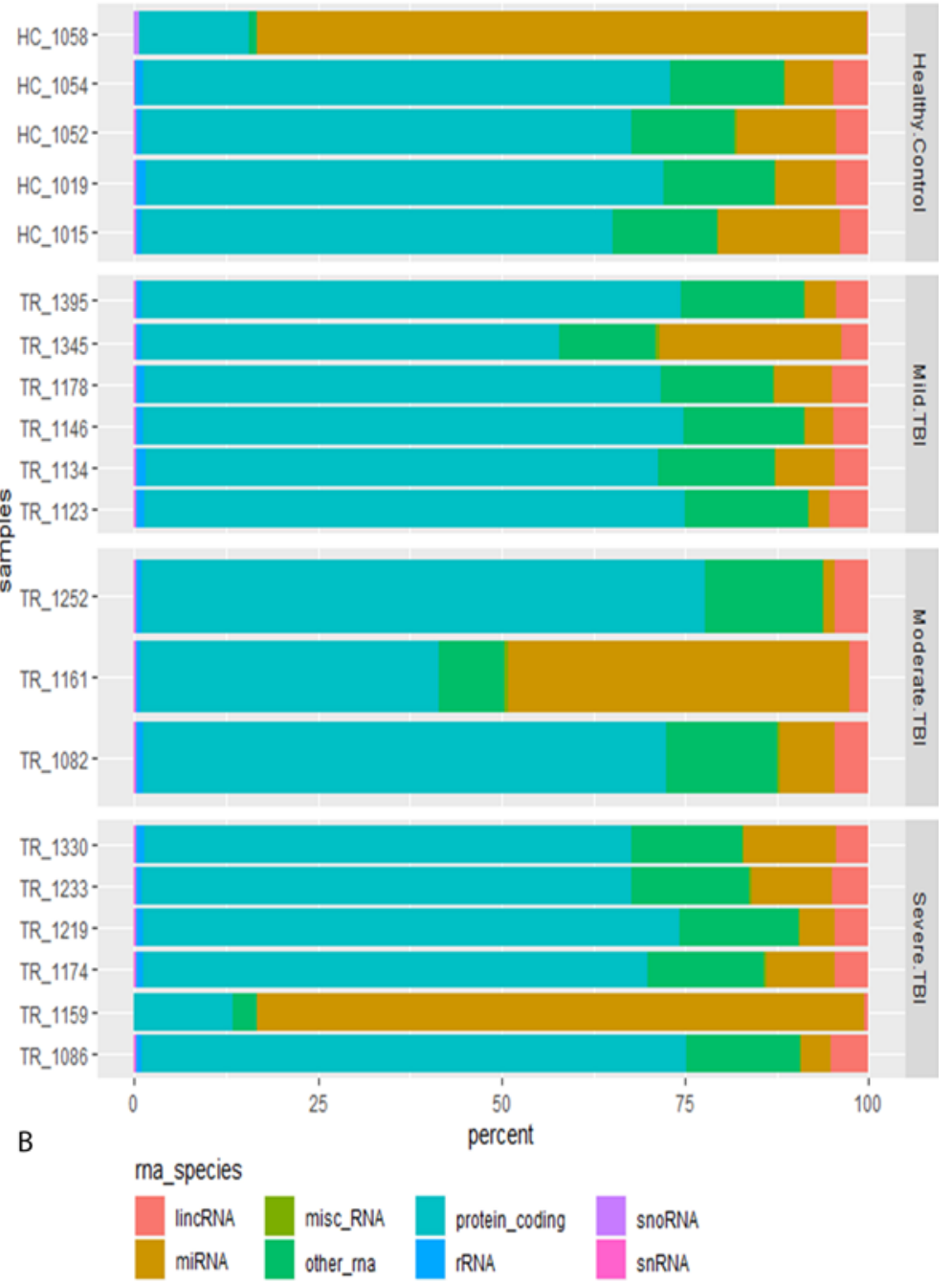

FIG. 3. Plasma exosomal RNA expression. A: Differential miRNA expression. Volcano plot of fold change versus $p$ value for miRNA sequences identified by short noncoding RNA sequencing of plasma EVs in TBI patients with altered consciousness (GCS score $\leq 14$ ) versus TBI patients and healthy controls with normal consciousness (GCS score $=15)$. Sequences trending to high differential expression (log 2 -fold change 0.7 and $p<0.1$ ) shown in red. B: Relative frequency of miRNA versus other RNA species in plasma exosomal RNA. of insult. It will also require a better understanding of how to isolate organ system-specific EVs to improve the signalto-noise ratio for studying biomarkers from these organ systems in response to injury. Lastly, the novel biomarkers themselves will have to be rigorously correlated with clinical presentations, disease states, and outcomes to ensure that the signatures in question truly are specific to the disease process and its treatment.

Assessing the biological significance of specific differentially expressed miRNA sequences identified by deep sequencing in this pilot study must be conducted cautiously, given our small patient sample sizes. Nevertheless, several intriguing possibilities can be observed. Although not directly implicated in TBI previously, miR-1 (increased in EVs from TBI patients with altered consciousness) upregulation has been reported in neurons undergoing apoptosis in response to hypoxia. ${ }^{15} \mathrm{miR}-143$ has been implicated in neuronal injury following ischemia and in regulating blood-brain barrier integrity. ${ }^{16}$ Finally, miR-9 (6.84 log 2-fold change increase in TBI with decreased consciousness, $\mathrm{p}<0.01$ in our data) has, in fact, been previously identified as a plasma extracellular miRNA biomarker in TBI in mice, although not in humans. ${ }^{17}$

\section{Limitations}

There are a number of limitations to this analysis, nearly all due to the small sample size, which includes multiple mechanisms of TBI, widely varying GCS scores at presentation, different patient demographics and characteristics, as well as only 5 healthy controls, and an initial sample size of only $500 \mu \mathrm{l}$ of plasma. These sample size limitations precluded the ability to perform reproducibility analyses and other standard validation techniques, potentially leading us to false conclusions from our pilot data. Despite the 
TABLE 3. Up- and downregulated miRNA sequences in patients with altered presentation GCS scores compared to normal presentation GCS scores

\begin{tabular}{ccc}
\hline miRNA Sequence & Log 2-Fold Change & $p$ Value \\
\hline Upregulated miRNA & & \\
\hline hsa-miR-1-3p & 1.51 & $<0.01$ \\
\hline hsa-miR-143-3p & 1.19 & $<0.01$ \\
\hline hsa-miR-151b & 3.71 & 0.09 \\
\hline hsa-miR-27a-3p & 0.89 & 0.02 \\
\hline hsa-miR-29a-3p & 1.37 & $<0.01$ \\
\hline hsa-miR-328-3p & 4.18 & 0.09 \\
\hline hsa-miR-9-3p & 6.84 & $<0.01$ \\
\hline hsa-miR-99a-5p & 1.32 & 0.05 \\
\hline hsa-miR-99b-5p & 1.15 & 0.06 \\
\hline Downregulated miRNA & & \\
\hline hsa-miR-155-5p & -2.99 & 0.03 \\
\hline hsa-miR-30c-5p & -1.24 & 0.09 \\
\hline
\end{tabular}

limitations of the cohort, significant differences were identified in GFAP concentration. Given the heterogeneous cohort, less stringent thresholds were required when analyzing the differential expression profiles of miRNA between groups, specifically a log fold change of 0.7 and a $p$ value $<$ 0.1 , resulting in the identification of 11 miRNA sequences that appear to differ between groups, but not all of which reach the threshold of $p \leq 0.05$. Given that this was a pilot analysis of 15 patients and 5 controls, a broad net was cast in order to identify any potential areas of interest that would warrant further analysis on a more stringent level. These specific miRNA sequences will be further analyzed in a more directed fashion with a much larger cohort and more stringent statistical analysis. The possibility of contamination of the RNA sequencing analysis must be considered, especially with such a small number of samples in this pilot study, and this furthers the need for larger studies to confirm or refute these findings.

We were also limited by the inability to completely isolate brain-specific EVs from systemic EVs in this analysis, and that likely somewhat decreased the signal-to-noise ratio for identification of TBI signatures. The significantly increased GFAP concentration in altered LOC patients would suggest that our bulk plasma EV samples were enriched for brain-derived EVs, but the actual proportion is unknown and could be quite small. We cannot state with complete certainty that GFAP is not simply a co-isolated contaminant in the ultracentrifugation process based on this analysis. Further studies will be required to identify how best to preserve the brain-specific EVs and discard the systemic EVs, after which differential expression analysis would likely reveal highly significant differences between groups. Nonetheless, this analysis provides a starting point to demonstrate that this analysis is feasible. Significant differences were quantifiable, even with a very small amount of plasma $(500 \mu \mathrm{l})$ from only 20 subjects with widely varying clinical and injury demographics. Further studies should attempt to isolate brain-specific EVs and analyze
TABLE 4. Top pathways for diseases and disorders or molecular and cellular functions targeted by differentially expressed plasma exosomal miRNAs, comparing TBI patients with altered consciousness (GCS scores $\leq 14$ ) and TBI patients and healthy controls with normal consciousness (GCS scores $=15$ )

\begin{tabular}{|c|c|c|}
\hline Variable & p Value Range & $\begin{array}{c}\text { No. of } \\
\text { Molecules }\end{array}$ \\
\hline \multicolumn{3}{|l|}{ Diseases \& disorders } \\
\hline Cancer & $4.75 \mathrm{E}-02$ to $1.35 \mathrm{E}-12$ & 10 \\
\hline Gastrointestinal disease & $3.84 \mathrm{E}-02$ to $1.35 \mathrm{E}-12$ & 10 \\
\hline $\begin{array}{l}\text { Organismal injury \& abnormali- } \\
\text { ties }\end{array}$ & $4.78 \mathrm{E}-02$ to $1.35 \mathrm{E}-12$ & 10 \\
\hline Respiratory disease & $8.49 \mathrm{E}-03$ to $1.35 \mathrm{E}-12$ & 8 \\
\hline Reproductive system disease & $3.43 \mathrm{E}-02$ to $1.00 \mathrm{E}-08$ & 9 \\
\hline \multicolumn{3}{|l|}{ Molecular \& cellular functions } \\
\hline Cell death \& survival & $4.13 \mathrm{E}-02$ to $8.72 \mathrm{E}-04$ & 6 \\
\hline $\begin{array}{l}\text { Cell-to-cell signaling \& interac- } \\
\text { tion }\end{array}$ & $1.97 \mathrm{E}-02$ to $9.05 \mathrm{E}-04$ & 4 \\
\hline $\begin{array}{l}\text { Cellular assembly \& organiza- } \\
\text { tion }\end{array}$ & $2.82 \mathrm{E}-02$ to $9.05 \mathrm{E}-04$ & 4 \\
\hline Cellular development & $4.22 \mathrm{E}-02$ to $9.05 \mathrm{E}-04$ & 9 \\
\hline $\begin{array}{l}\text { Nervous system development } \\
\text { \& function }\end{array}$ & $3.04 \mathrm{E}-02$ to $9.05 \mathrm{E}-04$ & 6 \\
\hline
\end{tabular}

them in much larger, uniform injury and presentation GCS score cohorts.

\section{Conclusions}

EVs can be isolated from peripheral blood after TBI, and they may contain brain-specific biomarkers that can be analyzed and potentially used in the diagnosis, prognosis, and treatment monitoring of TBI.

\section{Acknowledgments}

This study was funded in part by the Uihlein Scholarship from the Department of Neurological Surgery, Mayo Clinic, Rochester, MN.

\section{References}

1. Heijnen HF, Schiel AE, Fijnheer R, et al. Activated platelets release two types of membrane vesicles: microvesicles by surface shedding and exosomes derived from exocytosis of multivesicular bodies and alpha-granules. Blood. 1999;94(11): 3791-3799.

2. Kowal J, Tkach M, Théry C. Biogenesis and secretion of exosomes. Curr Opin Cell Biol. 2014;29:116-125.

3. Li CC, Eaton SA, Young PE, et al. Glioma microvesicles carry selectively packaged coding and non-coding RNAs which alter gene expression in recipient cells. RNA Biol. 2013;10(8): 1333-1344.

4. Proia P, Schiera G, Mineo M, et al. Astrocytes shed extracellular vesicles that contain fibroblast growth factor-2 and vascular endothelial growth factor. Int J Mol Med. 2008;21(1): 63-67.

5. Schiera G, Proia $P$, Alberti $C$, et al. Neurons produce FGF2 and VEGF and secrete them at least in part by shedding extracellular vesicles. J Cell Mol Med. 2007;11(6):1384-1394. 
6. Mathivanan S, Lim JW, Tauro BJ, et al. Proteomics analysis of A33 immunoaffinity-purified exosomes released from the human colon tumor cell line LIM1215 reveals a tissue-specific protein signature. Mol Cell Proteomics. 2010;9(2):197-208.

7. Korley FK, Kelen GD, Jones CM, Diaz-Arrastia R. Emergency department evaluation of traumatic brain injury in the United States, 2009-2010. J Head Trauma Rehabil. 2016; 31(6):379-387.

8. McMahon P, Hricik A, Yue JK, et al. Symptomatology and functional outcome in mild traumatic brain injury: results from the prospective TRACK-TBI study. J Neurotrauma. 2014;31(1):26-33.

9. Seabury SA, Gaudette É, Goldman DP, et al. Assessment of follow-up care after emergency department presentation for mild traumatic brain injury and concussion: results from the TRACK-TBI study. JAMA Netw Open. 2018;1(1):e180210.

10. Korley FK, Yue JK, Wilson DH, et al. Performance evaluation of a multiplex assay for simultaneous detection of four clinically relevant traumatic brain injury biomarkers. $\mathrm{J} \mathrm{Neu}$ rotrauma. 2018;36(1):182-187.

11. Manek R, Moghieb A, Yang Z, et al. Protein biomarkers and neuroproteomics characterization of microvesicles/exosomes from human cerebrospinal fluid following traumatic brain injury. Mol Neurobiol. 2018;55(7):6112-6128.

12. Okonkwo DO, Yue JK, Puccio AM, et al. GFAP-BDP as an acute diagnostic marker in traumatic brain injury: results from the prospective transforming research and clinical knowledge in traumatic brain injury study. J Neurotrauma. 2013;30(17):1490-1497.

13. Cumba Garcia LM, Peterson TE, Cepeda MA, et al. Isolation and analysis of plasma-derived exosomes in patients with glioma. Front Oncol. 2019;9:651.

14. Pitt JM, Kroemer G, Zitvogel L. Extracellular vesicles: masters of intercellular communication and potential clinical interventions. J Clin Invest. 2016;126(4):1139-1143.
15. Chang CY, Lui TN, Lin JW, et al. Roles of microRNA-1 in hypoxia-induced apoptotic insults to neuronal cells. Arch Toxicol. 2016;90(1):191-202.

16. Zeng X, Liu N, Zhang J, et al. Inhibition of miR-143 during ischemia cerebral injury protects neurones through recovery of the hexokinase 2-mediated glucose uptake. Biosci Rep. 2017;37(4):BSR20170216

17. Ko J, Hemphill M, Yang Z, et al. Multi-dimensional mapping of brain-derived extracellular vesicle microRNA biomarker for traumatic brain injury diagnostics. J Neurotrauma. Published online May 6, 2019. doi:10.1089/neu.2018.6220

\section{Disclosures}

The authors report no conflict of interest concerning the materials or methods used in this study or the findings specified in this paper.

\section{Author Contributions}

Conception and design: Puffer, Okonkwo, Parney. Acquisition of data: Puffer, Okonkwo, Parney. Analysis and interpretation of data: Puffer, Cumba Garcia, Himes. Drafting the article: Puffer, Cumba Garcia, Himes. Critically revising the article: Puffer, Cumba Garcia, Jung, Okonkwo, Parney. Reviewed submitted version of manuscript: Puffer, Okonkwo, Parney. Approved the final version of the manuscript on behalf of all authors: Puffer. Statistical analysis: Puffer, Cumba Garcia, Himes, Jung, Parney. Administrative/technical/material support: Meyer, Okonkwo. Study supervision: Okonkwo, Parney.

\section{Correspondence}

Ian F. Parney: Mayo Clinic, Rochester, MN. parney.ian@mayo. edu. 